\title{
Propuesta para enseñar inglés en preescolar a partir del análisis realizado en dos instituciones educativas de Santander
}

\begin{abstract}
Proposal to teach English in preschool from the analysis carried out in two educational institutions of Santander
\end{abstract}

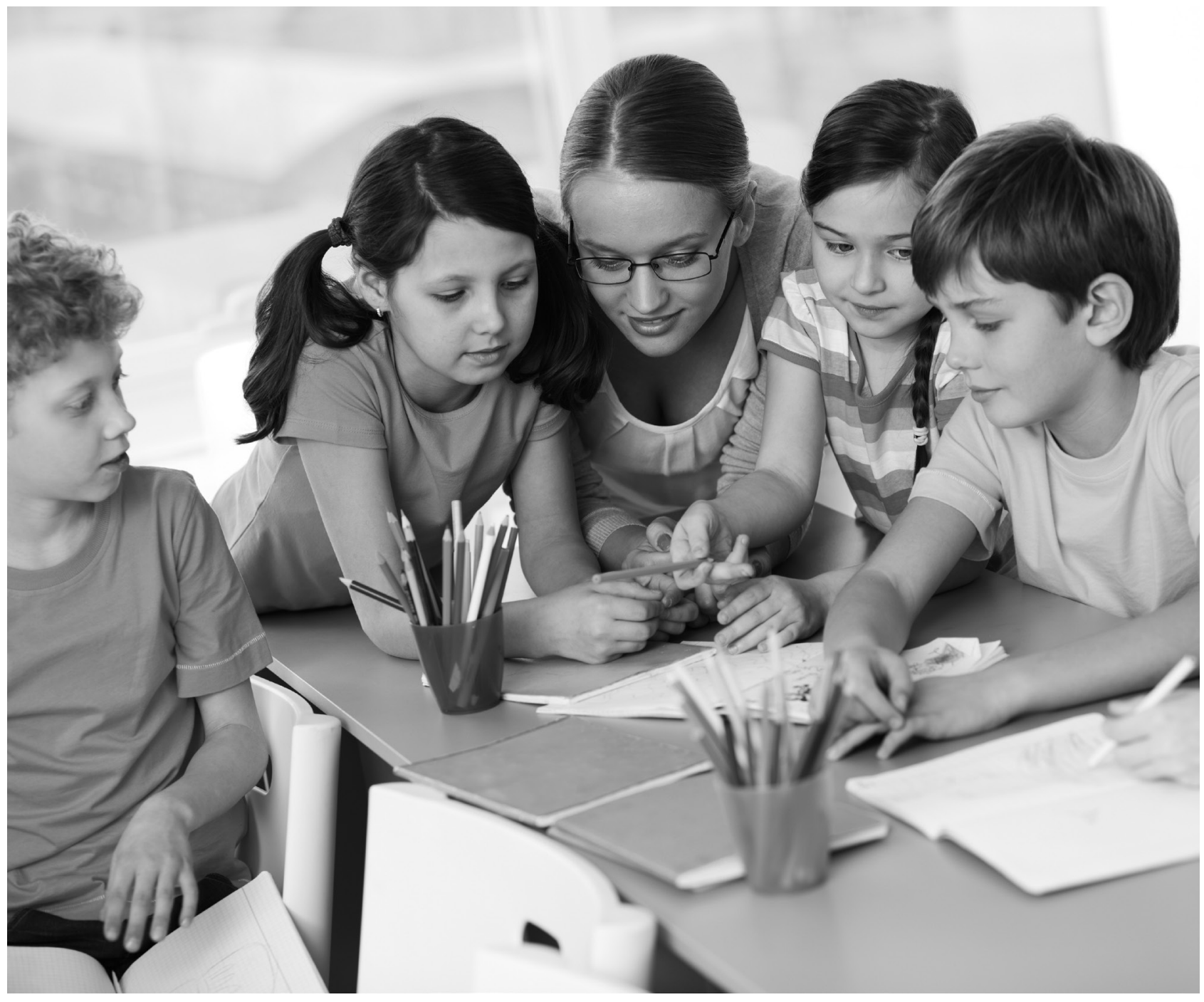




\title{
Propuesta para enseñar inglés en preescolar a partir del análisis realizado en dos instituciones educativas de Santander ${ }^{1}$ Proposal to teach English in preschool from the analysis carried out in two educational institutions of Santander
}

\author{
Miguel Fernando Pérez Calderón, ${ }^{2}$ Gustavo Alfonso Villamizar Acevedo ${ }^{3}$
}

Artículo recibido en agosto de 2017; artículo aceptado en febrero de 2018.

Este artículo puede compartirse bajo la Licencia Creative Commons Atribución-No Comercial-Compartir lgual 2.0 Genérica y se referencia usando el siguiente formato: Pérez, M. \& Villamizar, G. (2018). Propuesta para enseñar inglés en preescolar a partir del análisis realizado en dos instituciones educativas de Santander. I+D Revista de Investigaciones, 12 (2), 6-18.

DOI: https://doi.org/10.33304/revinv.v12n2-2018001

\section{Resumen}

La presente investigación, de carácter cualitativo, tuvo como objetivo analizar el proceso desarrollado para la enseñanza del inglés en el nivel de transición en dos instituciones educativas de Santander, para a partir de allí formular una propuesta de enseñanza. Para su cumplimiento se solicitó autorización de dos colegios ubicados en Floridablanca (Santander), uno público y el otro privado. Previo al aval se realizó una búsqueda bibliográfica que posibilitó la creación de cinco categorías deductivas y una inductiva. A partir de ella se diseñaron los instrumentos para recoger la información, los cuales se aplicaron a dos profesoras de transición, una del sector público y la otra del privado, que tienen a su cargo la enseñanza del inglés. Desde las categorías deductivas los datos recopilados permitieron dar cuenta de que ambas profesoras siguen una planeación, difieren en el valor que dan al manejo de la lengua materna por parte de los niños como insumo para aprender una segunda lengua, como estrategias de enseñanza corrigen constantemente la pronunciación y difieren en el uso de recursos tecnológicos. Como categoría inductiva emergió la relacionada con estrategias de enseñanza construida a partir del uso de recursos tecnológico y la lectura de historias. A partir del análisis de la información y como aporte a la enseñanza del inglés en preescolar se estructuró una propuesta didáctica.

Palabras clave: psicolingüística, enseñanza del inglés, preescolar, lengua extranjera, segunda lengua.

\footnotetext{
Abstract

This is a qualitative research which had as aim to analyze the English teaching process in transition level in two schools of Santander, from there to formulate a teaching proposal. To accomplish it, was requested authorization of the two

1. Artículo empírico, de enfoque cualitativo, resultado del proyecto de investigación para optar por el título de Magister en Educación, desarrollado en la Universidad Autónoma de Bucaramanga. Avenida 42 n. ${ }^{\circ} 48$ - 11. PBX 6436111 . Fecha de inicio: julio de 2015 - Fecha de terminación: diciembre de 2017.

2. Licenciado en Lengua Castellana e Inglés. Universidad Cooperativa de Colombia. Magister en Educación Universidad Autónoma de Bucaramanga (Colombia). Dirección: Avenida 42 n.o 48 - 11. PBX: 643 6111. ORCID ID: https://orcid.org/0000-0002-9389-4431. Correo electrónico: mperez27@ unab.edu.co.

3. Psicólogo, Universidad Nacional de Colombia. Doctor en Educación. Universidad Pedagógica Experimental Libertador (Venezuela), docente investigador del grupo Análisis y Transformación Psicosocial, Universidad Pontificia Bolivariana, Bucaramanga (Colombia). Dirección: Km 7 Autopista a Piedecuesta. PBX: 6796220, ext. 569. ORCID ID: https://orcid.org/0000-0002-8886-1993. Correo electrónico: gusvillamizar@yahoo.com.
} 
Miguel Fernando Pérez Calderón, Gustavo Alfonso Villamizar Acevedo

Propuesta para enseñar inglés en preescolar a partir del análisis realizado en dos instituciones educativas

de Santander

schools of Floridablanca (Santander), one of them public and the other private, before the endorsement, was done a bibliographic review which enables the creation of five deductive categories a one inductive. From there was designed the instruments to collect the information, these were applied to two transition teachers who taught English, one of a public school and the other of private. From deductive categories the collected data allows to show that the two teachers have a plan, they differ in the value of the mother language, of the children, as input to learn a second language. As principles of psycholinguistic they often correct the pronunciation mistakes and differ the use of the technological tools. As inductive category appeared teaching strategy built from the use of technological tools and stories. From the data analysis and as an input to the English teaching in preschool was structured a didactic proposal.

Keywords: psycholinguistic, English teaching, preschool, foreign language, second language.

\section{Introducción}

A pesar de que el Ministerio de Educación Nacional [MEN], promueve la enseñanza del inglés a temprana edad, no ha diseñado parámetros metodológicos para enseñar dicha lengua en el preescolar (Fandiño, Bermúdez \& Lugo, 2012), pero ello no ha sido óbice para que diversas instituciones ofrezcan cursos de inglés desde transición. El interés del MEN está en sintonía con la tendencia existente en países europeos que comienzan a enseñar inglés desde los seis años con la intención de desarrollar competencias de habla y escucha (Eurydice, 2012).

Esta situación lleva a preguntarse sobre cuándo empezar la enseñanza de un segundo idioma, la metodología que se debe seguir y el marco conceptual en el cual se debe fundamentar. Aunque un buen número de investigadores (Fleta, 2006; Kulh, 2010; Mayoral, 2016; Muñoz \& López, 2003; Navarro, 2010 \& Reyes, 2005) coinciden en que se debe enseñar a la edad más pronta posible, si bien no todos están de acuerdo sobre cómo y desde dónde hacerlo.

A partir del resultado de los trabajos presentados por diversos autores sobre el momento apropiado para aprender un segundo idioma, muchas instituciones educativas en el mundo han implementado el inglés como asignatura obligatoria en primaria; sin embargo, un gran número de estudiantes, a pesar de haber asistido regularmente a clases de inglés durante su educación formal, no adquieren el dominio esperado de la lengua (Puello \& Fernández, 2013).

Las razones que explican este fracaso son múltiples, entre ellas se encuentran: intensidad del tiempo empleada para la enseñanza, metodologías de enseñanza inadecuadas, pobreza de los recursos y deficiencias en el dominio de la lengua por parte de los profesores (Camelo, 2014). En lo concerniente a las competencias de los profesores para enseñar inglés, Pérez (2015) encontró que el $45,79 \%$ considera que está medianamente capacitado; el $32,71 \%$, que está muy capacitado y el $21,49 \%$ piensa que no está bien capacitado.
En lo que se refiere al uso de recursos, Méndez (2002) halló que el 53,77\% utiliza una gran variedad. Sobre el tiempo dedicado a la enseñanza del inglés, en la mayoría de los casos es de una hora semanal, por lo cual el 38,78\% considera que es medianamente suficiente, el 31,30\% que es suficiente y $29,90 \%$ que no es suficiente.

Sobre la metodología de enseñanza, Beke (2015) pudo ver que los maestros siguen impartiendo sus clases bajo el método tradicional, donde prima la enseñanza de la gramática sobre el desarrollo de habilidades comunicativas y creen que la buena pronunciación es un logro de la repetición.

Respecto a lo que sucede en Colombia, un reporte presentado por Education First English Profiency Index (2015) afirma que los colombianos poseen un bajo nivel de dominio del idioma inglés, situación que se evidencia a partir de los resultados alcanzados entre los setenta países que sometieron a evaluación, donde Colombia se ubicó en el puesto 57, aunque investigaciones del Ministerio de Educación Nacional (2015) muestran que el $2 \%$ de los estudiantes del grado $11^{\circ}$ poseen un nivel B1 de acuerdo con el Marco de Referencia Común Europeo en el dominio del idioma inglés.

Por otra parte, Santander ha obtenido mejores resultados en las pruebas Saber 11. En el 2010 el promedio en lengua extranjera fue de 46,9 y en el 2014 aumentó a 51,06 (Icfes, 2014). A pesar de esto, no se ha alcanzado lo propuesto en los estándares curriculares de la lengua, situación que lleva a preguntarse sobre la razón de ello, así como por las acciones que pueden llevar a su mejoramiento.

Además, es indispensable mencionar que aunque el inglés es una asignatura obligatoria en la educación básica y media, en el nivel de preescolar su enseñanza no está reglamentada. Por tanto, no existen lineamientos sobre cómo enseñar esa lengua en ese nivel, situación que llevó a la realización de esta investigación con el objetivo de analizar el proceso desarrollado para la enseñanza del inglés en el nivel de transición en dos instituciones educativas de Santander. A partir de allí 
se formula una propuesta de enseñanza con el fin de responder la siguiente pregunta: ¿qué propuesta pedagógica es pertinente para enseñar inglés en el nivel de transición en dos instituciones educativas de Santander, luego de verificar los procesos de enseñanza de esta lengua extranjera?

\section{Método}

\section{Tipo de estudio}

Este estudio está basado en el paradigma cualitativo, de carácter etnográfico.

\section{Tabla 1}

Características de las instituciones participantes

\section{Participantes}

La población objeto de este proyecto investigativo son dos docentes de preescolar del nivel de transición de dos instituciones educativas de la ciudad de Floridablanca (Santander), pertenecientes al estrato social 2, donde se enseña inglés a partir del nivel señalado.

Las instituciones donde se realizó la investigación se encuentran en barrios clasificados como estrato 2 y están ubicadas en la ciudad de Floridablanca. Las características de las dos instituciones se presentan en la Tabla 1.

\begin{tabular}{ccccc}
\hline Institución & Ubicación & Estrato & Escolaridad & Total, estudiantes \\
I1 & Lagos II & 2 & $\begin{array}{c}\text { Preescolar-Undécimo } \\
\text { grado }\end{array}$ & $\begin{array}{c}1400 \\
\text { transición }\end{array}$ \\
I2 & La Cumbre & 2 & Preescolar-Undécimo & 350 \\
\hline
\end{tabular}

Fuente: Autores

Los participantes fueron dos docentes, escogidos de manera no probabilística, por conveniencia, lo que, según Kinnear y Taylor (1989), puede obedecer al criterio del investigador. La selección de la muestra se dio por la asequibilidad a la información de los establecimientos.
De igual manera, la jornada de cada institución laboral brindaba facilidades a la hora de intervenir con los estudiantes o los docentes. Las características de las docentes participantes en la investigación se presentan en la Tabla 2.

\section{Tabla 2}

Características personales de las docentes participantes

\begin{tabular}{|c|c|c|c|c|c|}
\hline Docente & $\begin{array}{l}\text { Tipo de } \\
\text { colegio }\end{array}$ & Edad & Formación general & Formación en inglés & Experiencia \\
\hline 1 & Público & 57 años & Lic. En Primaria & 0 & 24 años \\
\hline
\end{tabular}

Fuente: Autores

\section{Materiales e instrumentos}

Con el fin de tener soportes suficientes al momento de hacer la interpretación y análisis de los datos, para su recolección se utilizaron las siguientes técnicas: observación, entrevista y revisión documental.

\section{Observación}

De acuerdo con Ander-Egg (2003), la observación hace referencia al proceso que se da para examinar y documentar hechos o momentos a través de los sentidos, y es definida como una forma natural de adquirir conocimiento día a día. Esta técnica también permite que se den procesos de ensayo y error, admitiendo a través de esto la creación de saberes.

En este estudio se realizó una observación no participante, directa y no sistematizada. Para llevarla a cabo se creó el instrumento Observación Clases de Inglés $(\mathrm{OCl})$. Lo observado se recopiló en un diario de campo y se tuvo en cuenta para observar el accionar en el aula de clase durante las sesiones de enseñanza de inglés, tanto del profesor como de los estudiantes. La observación 
se realizó en tres momentos: inicio, desarrollo y cierre, y fue efectuada por dos personas: el investigador $y$ otro docente, escogido y entrenado por el investigador para este proceso, los cuales se ubicaban en esquinas equidistantes para tener panorámicas diferentes.

Cada vez que se terminaba la sesión, los observadores anotaban el producto de este proceso en el diario de campo, el cual es una matriz que consta de tres entradas por dos. En ella registraban lo que acontecía en los tres momentos de la clase y las actividades que se desarrollaron en cada uno, así como los compartimentos de profesor y estudiantes y las interacciones entre ellos, y los recursos que utilizaban. En la Tabla 3 se presenta de manera sistematizada el proceso surtido en la observación.

Tabla 3

Proceso de observación

\begin{tabular}{|c|c|c|c|c|c|c|}
\hline $\begin{array}{c}\text { Cuestión que } \\
\text { observar }\end{array}$ & $\begin{array}{c}\text { Número de clases } \\
\text { observadas }\end{array}$ & Lugar & $\begin{array}{c}\text { Número de } \\
\text { observadores }\end{array}$ & Procedimiento & Sujetos & Registro \\
\hline $\begin{array}{l}\text { Clase de inglés, } \\
\text { durante tres } \\
\text { momentos: inicio, } \\
\text { desarrollo y cierre. } \\
\text { Acciones realizadas } \\
\text { por el docente y los } \\
\text { estudiantes. } \\
\text { Recursos utilizados } \\
\text { por el docente e } \\
\text { interacciones } \\
\text { docente-estudiante. }\end{array}$ & 10 & $\begin{array}{l}\text { Aula de clase, } \\
\text { dos instituciones } \\
\text { educativas, nivel } \\
\text { de transición. }\end{array}$ & 2 & $\begin{array}{l}\text { Cada observador } \\
\text { se ubica en } \\
\text { esquinas } \\
\text { equidistantes y } \\
\text { registra lo } \\
\text { observado en su } \\
\text { diario de campo, } \\
\text { luego comparten } \\
\text { lo registrado y se } \\
\text { hace un análisis } \\
\text { de lo registrado. }\end{array}$ & $\begin{array}{c}24 \text { estudiantes } \\
\text { en la I1 y } 11 \text { en } \\
\text { la I2 }\end{array}$ & $\begin{array}{l}\text { Diario de } \\
\text { campo. }\end{array}$ \\
\hline
\end{tabular}

Fuente: Autores

\section{Entrevista}

Otro instrumento que sirvió al desarrollo de este proceso fue la entrevista, la cual consiste, según Canales (como se citó en Díaz, Torruco, Martínez \& Varela, 2013, p. 163) en una "comunicación interpersonal establecida entre el investigador y el sujeto de estudio, a fin de obtener respuestas verbales a las interrogantes planteadas sobre el problema propuesto".

Para esta investigación, se realizó una entrevista semiestructurada, la cual, según Díaz et al (p. 163) "presenta un grado mayor de flexibilidad que las estructuradas, debido a que parte de preguntas planeadas, que pueden ajustarse a los entrevistados".

Para este proyecto se diseñó la Entrevista Semiestructurada Sobre Enseñanza del Inglés (ESEI), la cual constaba, según lo presupuestado por Ozonas y Pérez (2005), de una guía de entrevista con preguntas agrupadas de acuerdo con las categorías deductivas surgidas del análisis de las investigaciones leídas sobre el tema. La estructura de la entrevista se presenta en la Tabla 4.

\section{Tabla 4}

Estructura de la entrevista semiestructurada ESEI

\begin{tabular}{cl}
\hline Categorías & \multicolumn{1}{c}{ Preguntas } \\
\hline Planeación & 2. Está de acuerdo con la enseñanza de inglés a niños de preescolar. \\
& 3. ¿Qué elementos tiene en cuenta al planear la clase de inglés? \\
Saberes previos & 1. ¿Considera que es importante el dominio de la lengua materna por parte del estudiante para poder \\
& aprender inglés? \\
& 4. ¿Cuándo los preescolares cometen "errores en la pronunciación" los corrige de una vez? \\
& 5. ¿Cuándo los preescolares cometen "errores gramaticales" los corrige de una vez"? \\
& 6. ¿Al enseñar inglés enfatiza en habla, escucha, lectura o escritura?
\end{tabular}


Principios lingüísticos

Enseñanza comparada Recursos
7. ¿Al momento de enseñar inglés utiliza el español? Si la respuesta es afirmativa, ¿en qué situaciones?

9. ¿Utiliza mucha repetición?

10. ¿Empieza la enseñanza de una unidad con palabras o con frases?

11. ¿Durante la clase traduce de inglés a español?

12. ¿Al explicar en clase se ayuda con el lenguaje corporal?

13. ¿Existe diferencia en la manera como enseña inglés frente a las demás asignaturas?

8. ¿Utiliza recursos visuales variados para que los niños sepan el significado antes de usar la palabra?

Fuente: Autores

Esta entrevista es producto de una depuración previa hecha por dos expertos que evaluaron un instrumento que les fue entregado para que realizaran una revisión técnica.
La entrevista se realizó después de la aceptación de los entrevistados, y fue grabada y digitalizada. Los pasos seguidos para la elaboración y aplicación de la entrevista se sintetizan en la Tabla 5.

Tabla 5

Sistematización de la elaboración y aplicación de la entrevista

\begin{tabular}{|c|c|c|c|c|c|c|}
\hline $\begin{array}{c}\text { Revisión } \\
\text { teórica }\end{array}$ & $\begin{array}{l}\text { Categorías } \\
\text { deductivas }\end{array}$ & $\begin{array}{c}\text { Entrevista } \\
\text { inicial }\end{array}$ & $\begin{array}{l}\text { Evaluación } \\
\text { Expertos }\end{array}$ & $\begin{array}{l}\text { Entrevista } \\
\text { final }\end{array}$ & Aplicación & Análisis \\
\hline $\begin{array}{l}\text { Lectura sobre } \\
\text { el tema de } \\
\text { investigación }\end{array}$ & $\begin{array}{c}\text { Planeación } \\
\text { Saberes previos } \\
\text { Principios } \\
\text { lingüísticos } \\
\text { Enseñanza } \\
\text { comparada } \\
\text { Recursos }\end{array}$ & $\begin{array}{l}\text { Se elabora un } \\
\text { guion de } \\
\text { preguntas que se } \\
\text { sometió a juicio } \\
\text { de expertos. }\end{array}$ & $\begin{array}{l}\text { Dos expertos } \\
\text { evaluaron la } \\
\text { entrevista } \\
\text { siguiendo los } \\
\text { parámetros } \\
\text { que se les } \\
\text { entregaron }\end{array}$ & $\begin{array}{c}\text { Producto de lo } \\
\text { anterior, quedó } \\
\text { la entrevista } \\
\text { que se aplicó, } \\
\text { la cual consta } \\
\text { de } 13 \\
\text { preguntas. }\end{array}$ & $\begin{array}{c}\text { Previo } \\
\text { consentimiento, se } \\
\text { citó a las profesoras } \\
\text { en horas distintas a } \\
\text { su trabajo, en un } \\
\text { lugar apropiado. Sus } \\
\text { respuestas fueron } \\
\text { grabadas. }\end{array}$ & $\begin{array}{l}\text { Las respuestas } \\
\text { se analizaron } \\
\text { para dar } \\
\text { cumplimiento a } \\
\text { los objetivos. }\end{array}$ \\
\hline
\end{tabular}

Fuente: Autores

\section{Revisión documental}

La otra técnica que se usó fue la revisión documental. Según Hernández, Fernández y Baptista (2010), esta es una fuente muy valiosa ya que permite entender el fenómeno central del estudio. También, brinda la posibilidad al investigador de conocer antecedentes, situaciones y el funcionamiento cotidiano de algún contexto. Dicha revisión se implementó por medio de un cuadro que permitió contrastar toda la información de los documentos de clase con la teoría.

Finalmente, se hizo triangulación usando una tabla donde se hizo un análisis descriptivo entre las técnicas aplicadas en las dos instituciones para la enseñanza del inglés. Aquí fue evidente que las docentes emplean principios lingüísticos en la enseñanza del inglés al usar la repetición y el lenguaje corporal. Asimismo, ambas profesoras tienen una planeación estructurada. Frente al uso de recursos, la docente de la institución privada hace mayor uso de recursos tecnológicos. Finalmente, la docente del establecimiento privado les da gran importancia a los presaberes en la lengua materna para la enseñanza de una lengua extranjera, mientras que la otra docente dice que no es muy importante. Con esto, se dio paso a la construcción de una propuesta didáctica.

\section{Procedimiento}

De acuerdo con Pitman y Maxwell (como se citó en Rodríguez, Gil, \& García, 1996), toda investigación cualitativa requiere de un proceso continuo de decisiones y elecciones del investigador.

Se mencionan las fases del proyecto para así entender mejor como se desarrolló el estudio y cómo se llega al producto final.

Fase preparatoria. Inicialmente, se realizó la búsqueda de información en internet, bibliotecas y revistas. A partir de la revisión bibliográfica se crearon algunas categorías con las que se elaboraron los instrumentos a aplicar. Después, los instrumentos fueron sometidos a valoración de expertos y con base en lo sugerido se hicieron modificaciones. 
MIGUEL FERNANDO PÉREZ CALDERÓN, GUSTAVO ALFONSO VILLAMIZAR ACEVEDO

Propuesta para enseñar inglés en preescolar a partir del análisis realizado en dos instituciones educativas de Santander

Trabajo de campo. Primero fue necesario escoger las instituciones donde se llevó a cabo el estudio. Una vez seleccionadas, se habló con los directivos y se establecieron compromisos. Igualmente, se habló con las docentes participantes y se les explicó la aplicación de los instrumentos. Esta fase del proyecto se desarrolló a través de tres instrumentos. En primer lugar, se hizo una entrevista a las dos profesoras involucradas en este proceso con el fin de analizar su perspectiva acerca de la enseñanza del inglés. Asimismo, se hizo observación teniendo en cuenta un protocolo, mediante el cual se analizaron los pasos que se deben seguir en el momento de enseñar; a saber: inicio, desarrollo y cierre. Finalmente, se revisó el material que tenían las docentes para enseñar la lengua y la producción de los estudiantes. Previó a todo lo anterior las docentes participantes firmaron el consentimiento informado.

Fase analítica. Teniendo en cuenta la información recolectada con los protocolos, se identificó la manera como se enseña la lengua en la institución pública y en la institución privada. Después, se contrastó la información con la teoría para, finalmente, triangular los datos.

Fase informativa. En esta última fase se presentan los resultados obtenidos tras el proceso de recolección y análisis de datos. También se muestra el análisis del proceso de enseñanza del inglés en transición, y se hacen sugerencias al respecto. Igualmente, se ofrecen algunas recomendaciones para docentes de transición en los procesos de enseñanza del inglés y en el desarrollo de futuros proyectos de investigación.

\section{Resultados}

Se procede a exponer los hallazgos por categoría, mostrando cómo se logra cumplir con lo propuesto y se evidencian las fortalezas y debilidades del proceso de enseñanza del inglés de cada docente.

Para realizar la categorización fue necesario tener en cuenta lo que se observó en las clases, la revisión documental y las entrevistas. Se consideraron cinco categorías deductivas que surgen tras la revisión de objetivos y el análisis de la teoría leída. De la información producto del análisis de datos es posible que surjan categorías emergentes.

\section{Categorías deductivas}

Las categorías deducidas a partir del análisis de la teoría se presentan a continuación en la Figura 1.

Desde la categoría de planeación se evidenció que, al enseñar, las docentes tienen un orden establecido. Además, en los planes de área y clase se notó que la docente de la 12 no tiene en cuenta los lineamientos propuestos por el MEN para la enseñanza de las diferentes dimensiones del ser humano (García \& González, 2015).

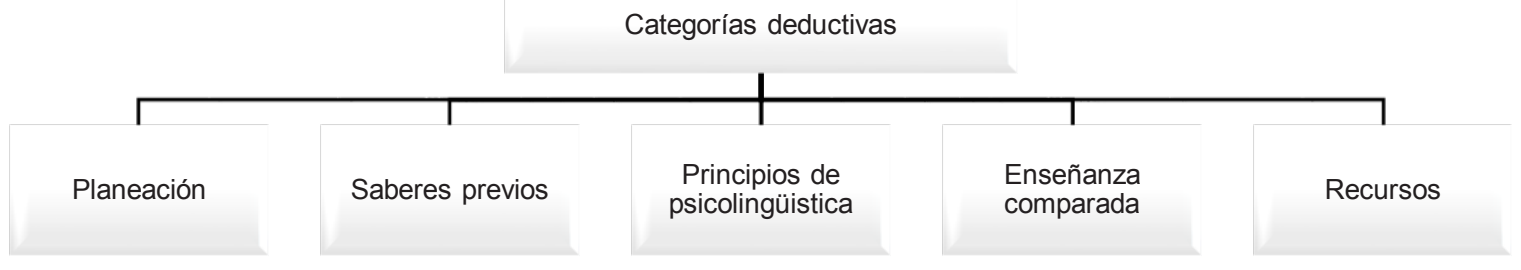

Figura 1. Categorías deducidas a partir del análisis de la teoría. Fuente: Autores

En la categoría de saberes previos se pudo notar que para la docente de la 12 es importante el dominio del español. Según ella, dominarlo le facilita al niño entender una lengua extranjera. Por el contrario, la docente de la 11 no da mucha importancia a ese hecho. Ella dice que a corta edad se pueden aprender al mismo tiempo varios idiomas, coincidiendo con lo presupuestado por Chomsky (como se citó en Silva, 2005) y Werker y Byers (2008), quienes afirmaron que se pueden aprender varios idiomas de forma simultánea.

En la categoría de principios de psicolingüística, la repetición del vocabulario es un procedimiento que las docentes ponen en práctica constantemente para corregir pronunciación. De igual manera, fue evidente que al referirse a cierto vocabulario las docentes hablaban en inglés el mayor tiempo posible, aunque la profesora de la 12 algunas veces presentó el vocabulario en español e inglés. Por ello se considera necesario que quien enseñe inglés domine el idioma y que la comunicación que se dé con los niños sea en dicho idioma, apoyándose en diferentes materiales para hacerse entender.

En la categoría de enseñanza comparada se pudo percibir que la docente de la 11 no establece muchas diferencias en la forma como planea las clases, mientras que la profesora de la 12 busca la transversalización al incluir otras competencias en las clases de inglés.

Por último, se analizó la categoría recursos. Se encontró que en la I1 la profesora diseña el material de clase, no 
hace uso de recursos tecnológicos, usa un big book en sus clases y diferentes materiales para la realización de manualidades. En la 12 se usa el video beam con sus parlantes. También, la docente diseña su propio material y se usa el recurso para manualidades en cada clase.

Valga anotar que el uso de recursos tecnológicos es muy importante, dado que genera impacto en los niños y facilita el aprendizaje. Esto lo sustentan Cadena y Leguizamón (2010), quienes mostraron cómo a través de actividades realizadas con Jclic se puede mejorar la habilidad del habla en inglés en estudiantes de prescolar.

Con base en lo anterior, se evidencia que es necesario usar diferentes recursos para que el niño tenga la oportunidad de aprender con diversidad de materiales, buscando que estos sean llamativos.

Como categoría inductiva se creó la de Estrategias de Enseñanza. Para ello se tuvo en cuenta el análisis hecho de las observaciones y la revisión documental. La docente de la I1 usaba un big book en las explicaciones. Como referente se tiene a Huennekens y Xu (2010), quienes demostraron el mejoramiento en habilidades lingüísticas en inglés usando un libro de historias. La docente de la 12 usaba constantemente canciones y material audiovisual. De acuerdo con un estudio hecho por Angarita (2010), con el uso de la música se logra mejorar el vocabulario en inglés de niños de prescolar, mostrando la diferencia de las clases tradicionales y las que tienen música, de lo cual se deduce que el uso adecuado de la música en los procesos de enseñanza de una lengua extranjera facilita el aprendizaje del niño. Asimismo, la lectura guiada de la manera correcta le permite al estudiante adquirir más vocabulario.

\section{Recomendaciones}

Teniendo en cuenta lo anterior, y tras revisar el trabajo de las docentes, se hacen algunas recomendaciones que surgen de este proceso investigativo con el fin de mejorar y enriquecer el proceso de enseñanza en inglés de docentes de prescolar y futuros proyectos de investigación. Igualmente, se presentan algunos aspectos a mejorar en este tipo de estudios. Las recomendaciones son:

Se propone que desde la dimensión comunicativa se haga una planeación que involucre el inglés y se den indicaciones sobre cómo trabajar una segunda lengua con niños de prescolar. Esto, atendiendo al hecho de que el MEN no tiene directrices relacionadas con la enseñanza del inglés en los primeros años de escolaridad.

Se sugiere a los docentes de transición crear actividades como saludar y dar nombre a los objetos del aula entre otras, que permitan a los estudiantes tener una comunicación en inglés de manera activa, tanto con sus compañeros como con el docente y el personal que pueda estar involucrado al proceso de enseñanza-aprendizaje.

Se plantea la importancia de la transversalización constante en los procesos de enseñanza del inglés con las demás asignaturas de prescolar, pues esto permite al estudiante fortalecer el aprendizaje del idioma y aprender nuevas temáticas.

Se sugiere a las instituciones educativas capacitar a los docentes a cargo de los primeros años de escolaridad en herramientas tecnológicas como videos, flash cards y juegos orientadas a la enseñanza del inglés en este nivel, para así lograr mayor impacto en lo que enseñan y por ende obtener mejores resultados. Involucrar procesos de lectura en inglés usando recursos tecnológicos puede permitir a los niños adquirir mayor vocabulario y entender mejor lo que se les explica.

Como eje central de las recomendaciones se plantea la siguiente propuesta para la enseñanza del inglés en preescolar, considerando que para desarrollar una propuesta didáctica para la enseñanza del inglés como lengua extranjera hay que tener en cuenta múltiples factores propios de los estudiantes, como los psicológicos, los lingüísticos y los cognitivos. En cuanto a los docentes, se debe considerar el dominio de la lengua, sus competencias pedagógicas y el manejo de los recursos.

Como esta propuesta se encuentra dirigida a docentes de preescolar, se centra en lo que se debe conocer y desarrollar en los estudiantes. Inicialmente, se aborda lo relacionado con los factores psicológicos, luego se pasa a las habilidades psicolingüísticas a desarrollar y en la tercera parte se presenta una sesión pedagógica.

\section{Factores psicológicos}

Estos tienen que ver con aspectos relacionados con el desarrollo cognitivo, lingüístico y neurológico del aprendiz, así como con su motivación e interés para aprender la lengua. Teniendo en cuenta que esta propuesta es para estudiantes de transición, la edad cronológica en la cual se encuentran oscila entre los cinco-seis años, que corresponde, de acuerdo con las etapas de desarrollo cognitivo propuesto por Piaget (1969), a la preoperacional. En esta etapa, el niño domina la gramática, y su lenguaje se extiende más allá de lo inmediato, tanto a nivel físico como temporal, debido al desarrollo del pensamiento simbólico y de la representación mental. Es decir, no se encuentra centrado en el aquí y el ahora. 
En esta etapa la capacidad imitativa está en su máximo nivel, pero no se queda en ella, dando paso a la creatividad. En el plano neurológico persiste la plasticidad cerebral. En los aspectos motivacionales muestra un claro interés por conocer e interactuar con personas de otras latitudes y culturas, lo cual forma parte de su curiosidad. Tiene una mayor tolerancia a la frustración y poco temor a hacer el ridículo. Los factores mencionados se sintetizan en la Figura 2.
En cuanto a los factores lingüísticos se considera que el niño entre los cinco y seis años ha adquirido el dominio de la lengua materna de un modo natural, espontáneo, sin responder a un programa de enseñanza, siempre y cuando su estructura mental y biológica sean óptimas. Para la adquisición de la lengua materna al niño le basta con interactuar con las personas de su entorno. El dominio de la lengua materna, que se considera fundamental para

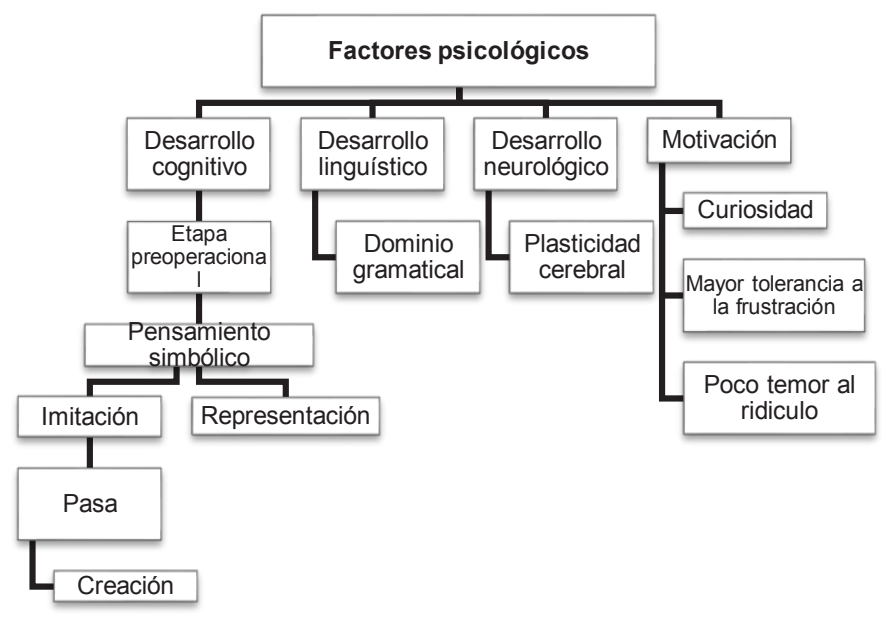

Figura 2. Síntesis de factores. Fuente: Autores

el aprendizaje de una segunda lengua, no se expresa en la reproducción mecánica de lo que el niño escucha sino en la creación de una gramática particular.

El niño en este momento ha desarrollado su competencia como oyente de su lengua materna, lo que le permite comprender órdenes, seguir instrucciones $y$, a su vez, emitir órdenes. En lo correspondiente al aprendizaje de una segunda lengua, se espera que comprenda lo que el profesor le enseña, así como las instrucciones que le da.

En cuanto a la producción, ésta también es de tipo verbal.
El niño debe reproducir los sonidos emitidos por el docente después que él los emita (imitación) y, luego, en una situación de la vida cotidiana que el profesor le señale. En la Figura 3 se sintetiza esta parte.

El tercer aspecto en consideración tiene que ver con los procesos cognitivos que intervienen en el aprendizaje de una segunda lengua, como son la percepción, la atención, la memoria y el razonamiento.

Desde la perspectiva psicolingüística se asume que para que la comprensión y la producción lingüística se den, es

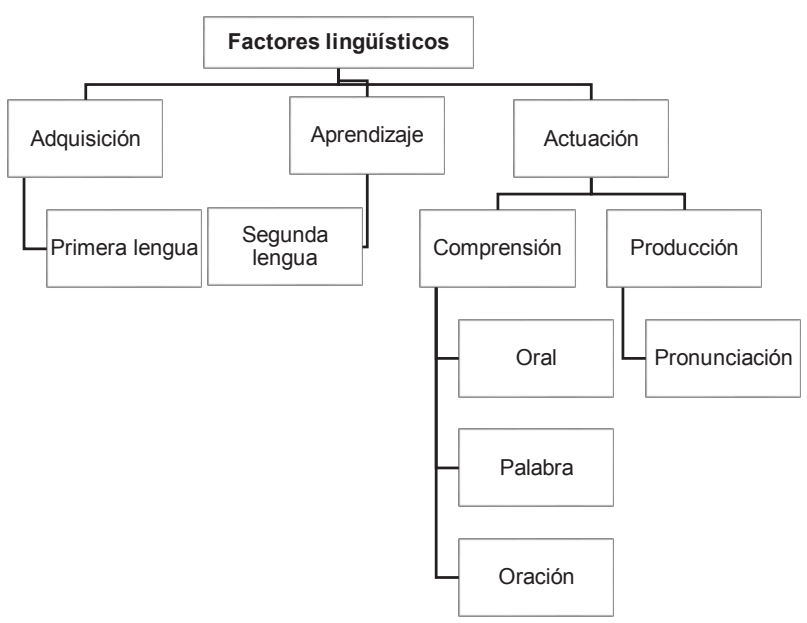

Figura 3. Factores lingüísticos. Fuente: Autores 
indispensable la participación de una serie de procesos cognitivos. A nivel perceptivo, teniendo en cuenta que el lenguaje es una serie de sonidos que el cerebro traduce y les da sentido, la percepción auditiva juega un papel importante, pues a partir de ella el niño identifica y relaciona el sonido con lo que denomina, ya sea a nivel representacional o en el plano concreto.

En cuanto a la atención, es necesario que el docente genere estrategias de enseñanza que lleven al niño a focalizarse en lo que está aprendiendo estas actividades deben ser variadas yágiles, considerando que la capacidad de focalización en un tema es relativamente corta.

Respecto a la memoria, vista como la capacidad del ser humano no solo de evocar información sino de actuar a partir de ella, uno de los aspectos a trabajar en el plano educativo tiene que ver con la retención de la información, pero no solo para repetirla sino para organizar cognitivamente lo aprendido. El profesor debe generar estrategias que le permitan al niño que la información que le da pase de la memoria a corto y a largo plazo.

Sobre el proceso de razonamiento se puede afirmar que es el pensamiento en acción, por lo que se asocia con la solución de problemas. El niño debe desarrollar habilidades cognitivas que le permitan enfrentar situaciones cotidianas de manera variada y exitosa. En la Figura 4 se sintetiza lo planteado.

\section{Habilidades psicolingüísticas}

Si bien la psicolingüística tiene entre sus objetivos analizar las actividades de comprensión y producción del lenguaje en los planos oral y escrito, debido a que esta es una propuesta para el nivel de transición, está centrada en la comprensión oral, se asume, como lo señala Frías (2002, p. 9), que "para que exista comprensión

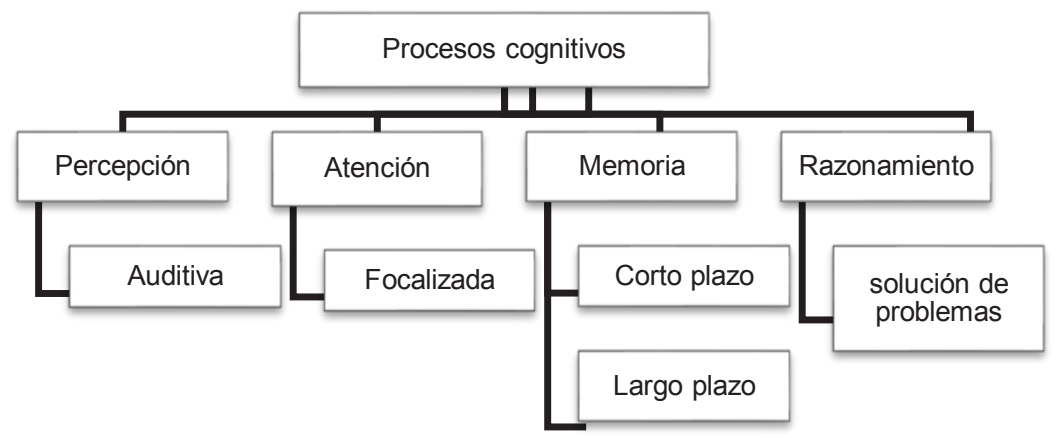

Figura 4. Procesos cognitivos. Fuente: Autores

es necesario que un sonido/grafía $X$ se identifique como perteneciente a un idioma $\mathrm{y}$, además, conocer el significado de X".

Memoria secuencial auditiva: Corresponde, según Kirk, McCarthy y Kirk (como se citó en Jara, Medina \& Meneses, 2010, p. 15) a "la habilidad para recordar y repetir series de material no significativo recién escuchadas, tales como secuencias de palabras, números, indicaciones".

Comprensión auditiva: Según Blanco, González, Ramírez, Torres y Valenzuela (como se citó en Jara, Medina \& Meneses, p. 15), tiene que ver con la "capacidad de obtener significado a partir de material presentado oralmente y relacionarlo con información conocida en una variedad de situaciones".

Comprensión visual: De acuerdo con Blanco et al (como se citó en Jara, Medina \& Meneses, p. 15), es la habilidad "para captar el significado de símbolos visuales".
Asociación auditiva: Según Blanco et al (como se citó en Jara, Medina \& Meneses, p. 15), es "la capacidad para relacionar conceptos presentados oralmente, por medio de oposiciones o analogías verbales".

Expresión motora (codificación motora): De acuerdo con Blanco et al (como se citó en Jara, Medina \& Meneses, p. $15)$, corresponde a la "capacidad para expresar ideas por medio de gestos corporales".

Expresión verbal: Para Blanco et al (como se citó en Jara, Medina \& Meneses, p. 16), corresponde a la "aptitud para expresar conceptos verbales (codificación vocal) y para nominar secuenciada y extensamente palabras pertenecientes al vocabulario activo o de uso diario".

Para desarrollar la propuesta se debe tener en cuenta que está enmarcada en las competencias que se espera lograr con el niño en el nivel de preescolar, en los planos cognitivo, afectivo y procedimental. En el nivel cognitivo se pretende que el niño aprenda a expresar y comprender 
MIGUEL FERNANDO PÉREZ CALDERÓN, GUSTAVO ALFONSO VILLAMIZAR ACEVEDO

Propuesta para enseñar inglés en preescolar a partir del análisis realizado en dos instituciones educativas

de Santander

términos que se usan en la comunicación cotidiana. En el procedimental, que desarrollen las competencias de escuchar y hablar. En el nivel actitudinal, que asuman y respeten normas y reglas de comportamiento. Estos propósitos no riñen con lo estipulado por el Ministerio de Educación Nacional (2010) en las Mallas de aprendizaje de inglés para transición a $5^{\circ}$ de primaria, que buscan desarrollar el aprestamiento para la escucha y para la conversación.

El logro de las competencias se alcanza con el trabajo conjunto de profesores, estudiantes y padres de familia; a su vez, la institución debe ofrecer los recursos necesarios.

Por ello se propone lo siguiente:

El maestro debe:

a. Hablar en inglés en el aula de clase y fuera de ella, y apoyar sus expresiones verbales con lenguaje corporal y gestual. El profesor debe hablar con buena pronunciación, usar las estructuras gramaticales de forma adecuada y estimular la creatividad del niño.

b. Repetir con frecuencia.

c. Evitar traducir en clase.

d. Promover la interacción entre los estudiantes, así como el trabajo colaborativo.

e. Las actividades deben estar centradas en el habla para desarrollar la capacidad de escucha mediante actividades lúdicas fundamentadas en la oralidad, como canciones, rondas, cuentos, poemas y otras manifestaciones de esta naturaleza.

f. Las actividades deben ser cortas y variadas para mantener la atención de los niños.

g. Practicar rutinas diarias.

h. Dejar de ser el centro de la acción pedagógica y tener en cuenta que el estudiante es el actor principal de este proceso.

Respecto a la planeación, se recomienda seleccionar actividades que despierten el interés del estudiante, que sean novedosas y variadas, que permitan alcanzar las metas establecidas y las competencias propuestas, así como relacionar lo conocido con lo nuevo.

Para la planeación hay que considerar varios momentos: antes de iniciar, iniciación, desarrollo y cierre.

Antes de iniciar se debe organizar el aula de clase y tener listos los materiales y recursos con los que se trabajará. Se despeja el centro del espacio, donde los niños se sentarán a escuchar las instrucciones iniciales y lo que el profesor considere indispensable informarles.

Iniciación: Al llegar los niños, el profesor los saluda en inglés; si se dan respuestas en español, el profesor las debe repetir en inglés. El profesor les pide a los niños que saluden a sus compañeros en inglés. Una vez centrada la atención, el profesor retoma elementos vistos en la clase anterior y pasa a explicar el tema que se va a desarrollar en la clase.

Desarrollo: Se da paso a las actividades programadas, que deben ser cortas y variadas, empleando los recursos adecuados para el tema, como canciones, juegos y videos. Se debe fomentar la participación de los niños por medio de demandas que no les generen presión.

Cierre: Antes de terminar la sesión el profesor debe repasar lo visto, haciendo evaluación oral, preguntando a cada niño sobre algunos de los aspectos vistos en clase; en este momento hace las correcciones pertinentes y se presenta un esbozo sobre la temática a desarrollar en la próxima sesión.

Entre los recursos, de la variedad y el material existentes, se pueden utilizar los siguientes:

a. Canciones y rondas infantiles.

b. Flashcards y posters.

c. Utilería para títeres y marionetas, desarrollando diálogos con ellos.

d. Hojas en blanco y colores para hacer dibujo libre que permita verificar el nivel de comprensión.

e. Juegos de memoria (tipo bingos) y de adivinar.

En cuanto a los temas para el desarrollo de los contenidos, se tiene en cuenta lo propuesto en diversos programas (Astudillo, 2015; Carbajal, 2015; Chacón, 2011; Rodao, 2010; Ministerio de Educación Nacional, 2010). En este sentido, se plantean dos grandes temas: el primero está relacionado con términos de uso cotidiano como días de la semana, meses del año, situaciones climáticas, saludos, partes del cuerpo, números cardinales, composición familiar, forma y tamaño de objetos, mascotas, alimentos, medios de transporte, lugares y servidores sociales.

El segundo con elementos lingüísticos, como el alfabeto, los pronombres, los artículos y las preposiciones de lugar. En lo relacionado con las unidades didácticas, Torres (1998) explica que son propuestas de trabajo que integran diversas áreas del conocimiento y se destinan a cubrir un periodo corto. Teniendo en cuenta lo que se busca enseñar en este estudio, los recursos que se usaron, la habilidad psicolingüística a desarrollar y la evaluación, en esta propuesta se presentará un ejemplo en la Tabla 6.

Unidad didáctica My family 
Tabla 6

Características personales de las docentes participantes

\begin{tabular}{|c|c|c|c|c|c|}
\hline Actividad & $\begin{array}{l}\text { Componente } \\
\text { comunicativo }\end{array}$ & $\begin{array}{c}\text { Habilidad } \\
\text { psicolingüística }\end{array}$ & Vocabulario & Recursos & Evaluación \\
\hline $\begin{array}{l}\text { El tema central es } \\
\text { la familia. } \\
\text { Utilizando } \\
\text { diversos recursos, } \\
\text { los niños } \\
\text { identificarán los } \\
\text { miembros de su } \\
\text { familia y los } \\
\text { nombrarán } \\
\text { teniendo en } \\
\text { cuenta su relación } \\
\text { con el grupo } \\
\text { familiar. }\end{array}$ & $\begin{array}{c}\text { Describe los } \\
\text { miembros de la } \\
\text { familia de acuerdo } \\
\text { con la denominación } \\
\text { que les corresponde. } \\
\text { Nombra a los } \\
\text { integrantes de su } \\
\text { familia. Identifica en } \\
\text { una figura los } \\
\text { miembros de una } \\
\text { familia. }\end{array}$ & $\begin{array}{l}\text { Comprensión auditiva. } \\
\text { Comprensión visual. } \\
\text { Expresión verbal }\end{array}$ & $\begin{array}{l}\text { Mother, father, } \\
\text { grandfather, } \\
\text { grandmother, } \\
\text { brother, sister, } \\
\text { aunt, uncle. }\end{array}$ & $\begin{array}{l}\text { Flashcards, } \\
\text { Hojas de papel, } \\
\text { Lápices de } \\
\text { colores, Fotos } \\
\text { del álbum } \\
\text { familiar. }\end{array}$ & $\begin{array}{l}\text { Para demostrar su } \\
\text { aprendizaje, cada niño } \\
\text { dibujará su familia e } \\
\text { identificará sus } \\
\text { miembros utilizando el } \\
\text { vocabulario aprendido } \\
\text { en clase. }\end{array}$ \\
\hline
\end{tabular}

Fuente: Autores

\section{Referencias}

Ander-Egg, E. (2003). Métodos y técnicas de investigación social IV, técnicas para la recogida de datos $e$ información. Buenos Aires: Lumen Humanitas.

Angarita, N. (2010). Las canciones en inglés como recurso pedagógico para el mejoramiento de la pronunciación de la lengua inglesa en los estudiantes del grado noveno del Colegio San Fernando de la ciudad de Valledupar. (Trabajo de grado para optar el título de Especialista en Educación). Universidad Mariana, Valledupar (Colombia).

Astudillo, S. (2015). Diseño de una unidad didáctica para la enseñanza del inglés como lengua extranjera utilizando el storytelling para el desarrollo de la competencia comunicativa y del pensamiento crítico en niños degrado transición (Tesis de pregrado). Universidad del Valle, Cali (Colombia). Recuperado de http://bibliotecadigital. univalle.edu.co/bitstream/10893/9043/1/CB-0434001.pdf

Beke, R. (2015). Inglés en la educación secundaria pública en Venezuela. En E. Zeuch \& M. Gregson (Coords.). La enseñanza del inglés en la escuela pública venezolana: evidencias, experiencias y perspectivas (47-76). Caracas: British Council.

Cadena, M. \& Lequizamón, Y. (2010). Improving spelling skills in kindergardeners through Jclic activities (Tesis de maestría). Universidad de la Sabana. Chía (Colombia). Recuperado de https://intellectum. unisabana.edu.co/handle/10818/3194

Camelo, G. (2014). Modelo de formación del profesorado en tecnología educativa. I+ D Revista de Investigaciones, 3(1), 18-23. Recuperado de https:// doi.org/10.33304/revinv.v03n1-2014002

Carbajal, R. (2015). Guía para el docente de la enseñanza del inglés como segunda lengua. Realidad y Reflexión,
$15(41), 43-57$.

Chacón, M. (2011). Material Complementario para el curso Inglés para Educadores de Preescolar. Madrid: UNED.

Díaz, L., Torrico, U., Martínez, M. \& Varela, M. (2013). La entrevista, recurso flexible y dinámico. Investigación Educación Médica, 2 (7), 163.

Education First English Profiency Index. (2015). Estudio de Dominio de Inglés en adultos para América Latina. Recuperado de https://www.ef.com.es/about-us/ highlights/2015/ef-epi-release-2015/

Eurydice. (2012). Cifras claves de la enseña de lenguas en los centros escolares de Europa. Madrid: EACEA.

Fandiño, Y., Bermúdez, J. \& Lugo, V. (2012). Retos del Programa Nacional de Bilingüismo. Colombia Bilingüe. Educación y Educadores, 15(3), 363-382.

Fleta, M. (2006). Aprendizaje y técnicas de enseñanza del inglés en la escuela. Encuentro, 16, 51-62.

Frías, X. (2002). Introducción a la psicolingüistica. Recuperado de www.sld.cu/galerias/pdf/ sitios/rehabilitacion-logo/introduccion_a_la_ psicolinguistica.pdf

García, E. \& González, I. (2015). La universidad educa para la libertad. Reflexiones de un humanismo personalista. I+ D Revista de Investigaciones, 5(1), 108123. Recuperado de https://doi.org/10.33304/revinv. v05n1-2015007

Hernández, R., Fernández, C. \& Baptista, M. (2010). Metodología de la investigación. Mexico D.F: Mc Graw-Hill.

Huennekens, M. \& Xu, Y. (2010). Effects of a CrossLinguistic Storybook Intervention on the Second Language Development of Two Preschool English Language Learners. Early Childhood Education Journal, 38, 19-26.

Instituto Colombiano para el Fomento de la Educación 
MIGUEL FERNANDO PÉREZ CALDERÓN, GUSTAVO ALFONSO VILLAMIZAR ACEVEDO

Propuesta para enseñar inglés en preescolar a partir del análisis realizado en dos instituciones educativas

de Santander

Superior. (2014). Reportes Históricos. Recuperado de http://www.icfesinteractivo.gov.co/historicos/

Jara, P., Medina, E. \& Meneses, M. (2010). Perfil de habilidades psicolingüisticas y lingüísticas en niños de 4 a 5.11 años con antecedente de prematurez extrema. (Tesis de pregrado). Universidad de Chile, Santiago de Chile. Recuperado de http://repositorio.uchile.cl/ handle/2250/114159

Kinnear, T. \& Taylor, J. (1989). Investigación de mercados. Un enfoque aplicado. Colombia: McGraw-Hill.

Kuhl, P. (2010). Brain mechanisms in early language acquisition. Neuron, 67, 713-727.

Mayoral, P. (2016). Estrategias didácticas para la enseñanza del idioma inglés a niños de preescolar: el caso de un colegio en Colima, México. (Tesis de doctorado). Instituto Tecnológico y de Estudios Superiores de Occidente, México. Recuperado de https://rei.iteso. $\mathrm{mx} /$ handle/11117/3790

Méndez, G. (2002). Formación de profesores de inglés de escuelas preescolares y primarias. (Tesis de maestría). Universidad de Colima. México. Recuperado de http://digeset.ucol.mx/tesis_posgrado/Pdf/ Guadalupe\%20Socorro\%20Mendez\%20Flores.pdf

Ministerio de Educación Nacional. (2015). "Dominio del inglés nos permite aumentar la inversión extranjera y las oportunidades", Ministra de Educación. Recuperado de http://www.mineducacion.gov.co/ cvn/1665/w3-article-354233.html

Ministerio de Educación Nacional. (2010). Serie lineamientos curriculares preescolar. Bogotá: Ministerio de Educación Nacional.

Muñoz, C. \& López, D. (2003). Aprendizaje temprano de una segunda lengua. Encuentro, 13(14), 169-175.

Navarro, B. (2010). Adquisición de la primera y segunda lengua en aprendientes en edad infantil y adulta. Revista Semestral de Iniciación a la Investigación en Filología. 2, 115-128.

Ozonas, L.\&Pérez,A.(2005) Laentrevistasemiestructurada. Notas sobre una práctica metodológica desde una perspectiva de género. La Aljaba. 9,198-203.

Pérez, T. (2015). Inglés en el sector público: la situación actual en las escuelas primarias de Venezuela. En E. Zeuch \& M. Gregson (coords.). La enseñanza del inglés en la escuela pública venezolana: evidencias, experiencias y perspectivas (13-46). Caracas: British Council.

Piaget, J. (1969). Seis estudios de psicología. Barcelona: Planeta.

Puello, D. \& Fernández, M. (2013). Sistema para la detección de estilos de aprendizaje. I+ D Revista de Investigaciones, 1(2), 42-49. Recuperado de https:// doi.org/10.33304/revinv.v02n2-2013004

Reyes, E. (2005). El aprendizaje de una lengua extranjera en el aula de educación infantil. Recuperado de http:// aulainfantil.grao.com/revistas/aula-infantil/27, 17-18

Rodao, E. (2010). Hacia el diseño de una propuesta pedagógica para la enseñanza de inglés a niños de 3 a 5 años del centro de desarrollo integral: "Semillas de Vida".(Tesis de pregrado). Pontificia Universidad Javeriana. Bogotá (Colombia). Recuperado de repository.javeriana.edu.co/ bitstream/handle/10554/5922/tesis700

Rodríguez, G., Gil, J. \& Garcia, E. (1996). Metodología de la investigación cualitativa. Granada: Aljibe.

Silva, O. (2005). ¿Hacia dónde va la lingüística? Forma y función, 18, 229-249.

Torres, J. (1998). Elaboración de unidades didácticas integrales. Globalización e interdisciplinariedad: el curriculum integrado (pp. 220-264). Madrid: Morata.

Werker, J. \& Byers, K. (2008). Bilingualism in infancy; first steps in perception and comprehension. Trends in Cognitive Sciences, 12 (4), 144-15. 